\title{
Valoración social económica ambiental de los incendios forestales
}

\author{
Social economic environmental valuation of forest fires
}

\section{Valoração socioeconômica ambiental de incêndios florestais}

\author{
Jaime Cueva Toalombo ${ }^{1}$ \\ rena_cuajismh@hotmail.com \\ https://orcid.org/0000-0003-4677-7643
}

\author{
Elvia Caiza Criollo² \\ marinac2083@gmail.com \\ https://orcid.org/0000-0002-0377-5798
}

\author{
Marita Salguero ${ }^{1}$ \\ salgueromaritza@yahoo.com \\ https://orcid.org/0000-0002-8500-016X
}

\author{
${ }^{1}$ Universidad Técnica de Ambato-Ambato, Ecuador \\ ${ }^{2}$ Universidad Técnica de Cotopaxi-Latacunga, Ecuador
}

\section{RESUMEN}

En la investigación desarrollada sobre la valoración socio económica ambiental de los incendios forestales, en la provincia de Imbabura, se realizó una valoración económica ambiental utilizando la metodología proporcionada por el Ministerio Ambiental con plantaciones forestales de Eucalyptus globulus; a través de un estudio se logró determinar que los bosques andinos nativos presentan un valor económico ambiental de 77783,4 USD/ha, el mismo que es superior al de las plantaciones forestales que tienen 46344,1 USD/ha y 45471,9 USD/ha. Por otro lado, también se encontró que la producción de biomasa es netamente superior en las plantaciones forestales que, en los bosques nativos (E. globulus 492,59 ton/ha, P. patula 333,8 ton/ha y Bosques nativos 151,91 ton/ha), con cantidades que superan más del $50 \%$ y que, además se ve reflejado en el almacenamiento de carbono, ya que las plantaciones forestales al tener mayor producción de biomasa, también retinen mayor cantidad de carbono ( $E$. globulus con 246,30 ton/ha, P. patula con 166,93 Bosque y andino 75,96 ton/ ha).

Palabras clave: Incendio Forestal; Valoración Socio Económica; Clima; Ambiental; Biomasa; Carbono; Boque; Eucalyptus Globulos

\section{ABSTRACT}

In the research carried out on the socioeconomic environmental assessment of environmental fires, in the province of Imbabura, an economic assessment was carried out using the methodology provided by the Ministry of the Environment with forest plantations of Eucalyptus globulus. Through a study it was possible to determine that the forests and natives have an environmental economic value of 77783.4 USD / ha, the same that is higher than that of the forest plantations that have 46344.1 USD / ha and 45471.9 USD / say ah. On the other hand, it was also found that biomass production is clearly higher in forest plantations than in native forests (E. globulus 492.59 ton / ha, P. patula 333.8 ton / ha and native forests 151, 91 ton / ha), with amounts that exceed more than $50 \%$ and that, in addition, is reflected in carbon storage, since forest plantations have higher biomass production, also retinal a greater amount of carbon (E. globulus with 246.30 ton / ha, P. patula with 166.93 Forest and Andean 75.96 ton / ha).

Key words: Forest Fire; Socio-Economic Assessment; Climate; Environmental; Biomass; Carbon; forest; Eucalyptus Globulos

\section{RESUMO}

$\mathrm{Na}$ pesquisa realizada sobre a avaliação socioeconômica ambiental de incêndios florestais, na província de Imbabura, foi realizada uma avaliação ambiental econômica utilizando a metodologia fornecida pelo Ministério do Meio Ambiente com plantações florestais de Eucalyptus globulus; Através de um estudo, foi possível determinar que as florestas nativas andinas possuem um valor econômico ambiental de 77.783,4 USD/ha, o mesmo que é superior ao das plantações florestais que possuem 46.344,1 USD/ha e 45.471,9 USD/ha. Por outro lado, verificou-se também que a produção de biomassa é claramente maior nas plantaçōes florestais do que nas florestas nativas (E. globulus 492,59 ton/ha, P. patula 333,8 ton/ha e florestas nativas 151, 91 ton/ha), com quantidades que ultrapassam mais de $50 \%$ e que, além disso, se reflete no armazenamento de carbono, uma vez que as plantações florestais têm maior produção de biomassa, também retinal uma maior quantidade de carbono (E. globulus com 246,30 ton/ha, P. patula com 166,93 e andina 75,96 ton/ha).

Palavras-chave: Incêndio Florestal; Avaliação Socioeconômica; Clima; Ambiental; Biomassa; Carbono; floresta; Eucalyptus Globulos 


\section{INTRODUCCIÓN}

Los daños causados por los incendios forestales han aumentado en las últimas décadas en todo el mundo (1). En estos marcos, la comunidad científica puede y debe aportar contribuciones, concretamente a través de nuevos enfoques y conocimientos que permitan prevenir las consecuencias negativas de los incendios forestales $y$ permitan comprender los diversos impactos después de su ocurrencia. Las metodologías actuales para valoración de los efectos de los incendios forestales presentan una modelización matemática y computacional, aporta nuevos enfoques para la evaluación de incendios forestales ya que permite vincular varias dimensiones relacionadas con estas realidades multidisciplinarias, tomando en cuenta el máximo potencial generado por las áreas verdes.

Las evaluaciones de los impactos de los incendios forestales son contribuciones importantes al diseño de políticas agroforestales ajustadas, específicamente con el fin de dirigir el apoyo a las actividades más afectadas negativamente. Los instrumentos de política y la legislación relacionada son herramientas determinantes para reducir los impactos negativos de los incendios forestales (2).

De hecho, las tierras agroforestales dentro y fuera de Ecuador continúan ardiendo, en algunos casos con mayor severidad $y$ frecuencia, principalmente debido al cambio climático y al calentamiento global.

Por otro lado, los gobiernos y las instituciones relacionadas crean (y divulgan) más regulaciones que en el pasado. Quizás, se necesiten nuevos enfoques, donde las nuevas tecnologías puedan jugar un papel determinante. Los enfoques multidisciplinarios podrían aportar contribuciones interesantes, por ejemplo, el análisis económico podría transmitir conocimientos valiosos. Los incendios forestales siempre han tenido implicaciones negativas; sin embargo, los impactos de los incendios forestales no siempre son iguales en los distintos sectores económicos (3). Estas realidades exigen más aspectos destacados sobre las consecuencias reales de los incendios forestales en las diferentes actividades económicas.

\section{MATERIALES Y METÓdOS}

Las herramientas y equipos que se utilizó para la toma de datos en el campo son: cinta diamétrica (medir diámetro), hipsómetro suunto (medir altura de árboles), podadora de aérea y de mano, segueta y balanza analógica. Además, para realizar el proceso de información y toma de datos en el laboratorio, se usó balanzas electrónicas de precisión y una estufa, la cual fue utilizada para secar las muestras.

\section{Determinación del valor económico ambiental de los servicios y bienes del bosque nativo andino y plantaciones forestales (Pinus patula y Eucalyptus globulus).}

Con la finalidad de obtener el valor económico ambiental de los tres tipos de cobertura, se trabajó con la metodología propuesta por el Ministerio del Ambiente del Ecuador, la misma que está basada en los servicios y bienes que proporcionan los ecosistemas (4). 


\section{Belleza escénica}

Para determinar el costo de la belleza escénica, se tomó como referencia las visitas al Parque Nacional Podocarpus en el sector Cajanuma (ubicado cerca de la zona del sector de Uritusinga), que según estadísticos del MAE (Ministerio del Ambiente) en el año 2017 ingresaron 9475 turistas de los cuales 7379 son nacionales y 2078 extranjeros. Además, se aplicó una encuesta a 30 turistas nacionales y extranjeros sobre su disposición a pagar, la misma que fue realizada en el puesto de control de ingreso al área.

El resultado obtenido de la encuesta, facilito la obtención de datos referentes al costo que los turistas nacionales y extranjeros estarían dispuestos a pagar, para conocer las áreas turísticas.

\section{Medición de las variables de árboles en pie y toma de muestras}

En primer lugar, se midió el diámetro del árbol a la altura de pecho (DAP), la altura comercial $(\mathrm{Hc})$ y altura total del fuste $(\mathrm{Ht})$. El DAP se midió con cinta diamétrica, utilizando como herramienta de precisión una barra de 1,30 m de largo. La altura total y comercial se midió con hipsómetro suunto.

\section{Determinación del número de unidades de muestreo a medir por estrato}

La determinación de la biomasa se la realizó en los diferentes compartimentos del bosque. Para el estrato arbóreo se midió individuos mayores a $10 \mathrm{~cm}$ de DAP, dentro de ellos, para el estrato arbustivo, se estableció dos parcelas una de $5 \times 5 \mathrm{~m}$ y otra de $2 \times 2 \mathrm{~m}$ para el estrato Herbáceo y para el caso de Necromasa se estableció solo una de $2 \times 2 \mathrm{~m}$.

\section{Biomasa arbórea}

Dentro de la superficie de cada parcela se midió el DAP de 1,30 m del fuste (con lo cual se puede calcular el área basal que comprende la zona de cualquier sección transversal del fuste de un árbol, y la altura del fuste de cada árbol.

\section{RESULTADOS Y DISCUSIÓN}

En la Figura 1 se representa el número de incendios forestales registrados en la provincia de Imbabura generados en la última década (2010-2019), así como también el área afectada en zonas arboladas y no arboladas mostrado en la figura 8 . Se puede evidenciar que en el año 2015 se registró el mayor número de incendios forestales con 841 eventos, mientras que en el año 2012 el área afectada fue mayor en comparación a los últimos años. Cabe mencionar que la mayor incidencia de los registros obtenidos se encuentra en el cantón de Cotacachi, así como también el número de incendios no es directamente proporcional a la superficie afectada (5). 


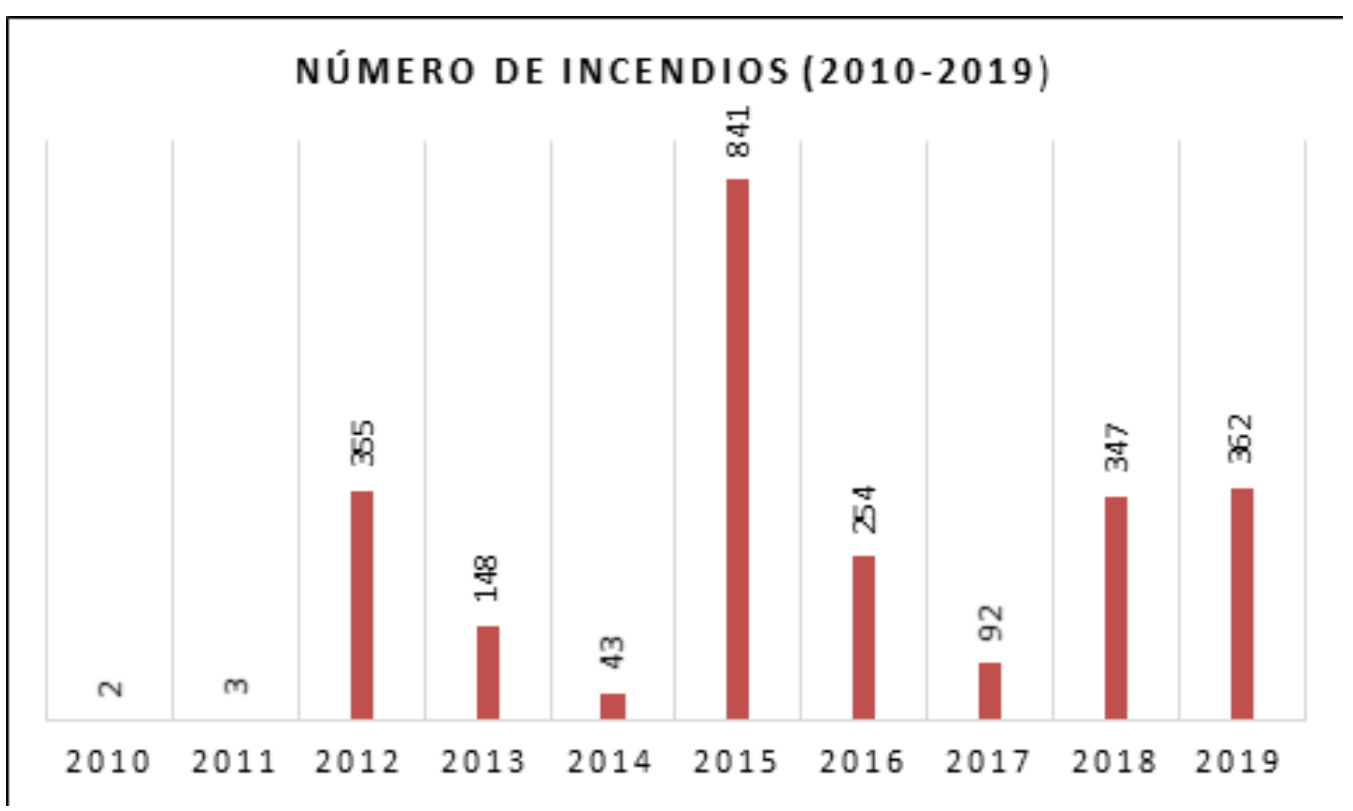

Figura 1. Registro de número de incendios en la última década. Elaboración propia.

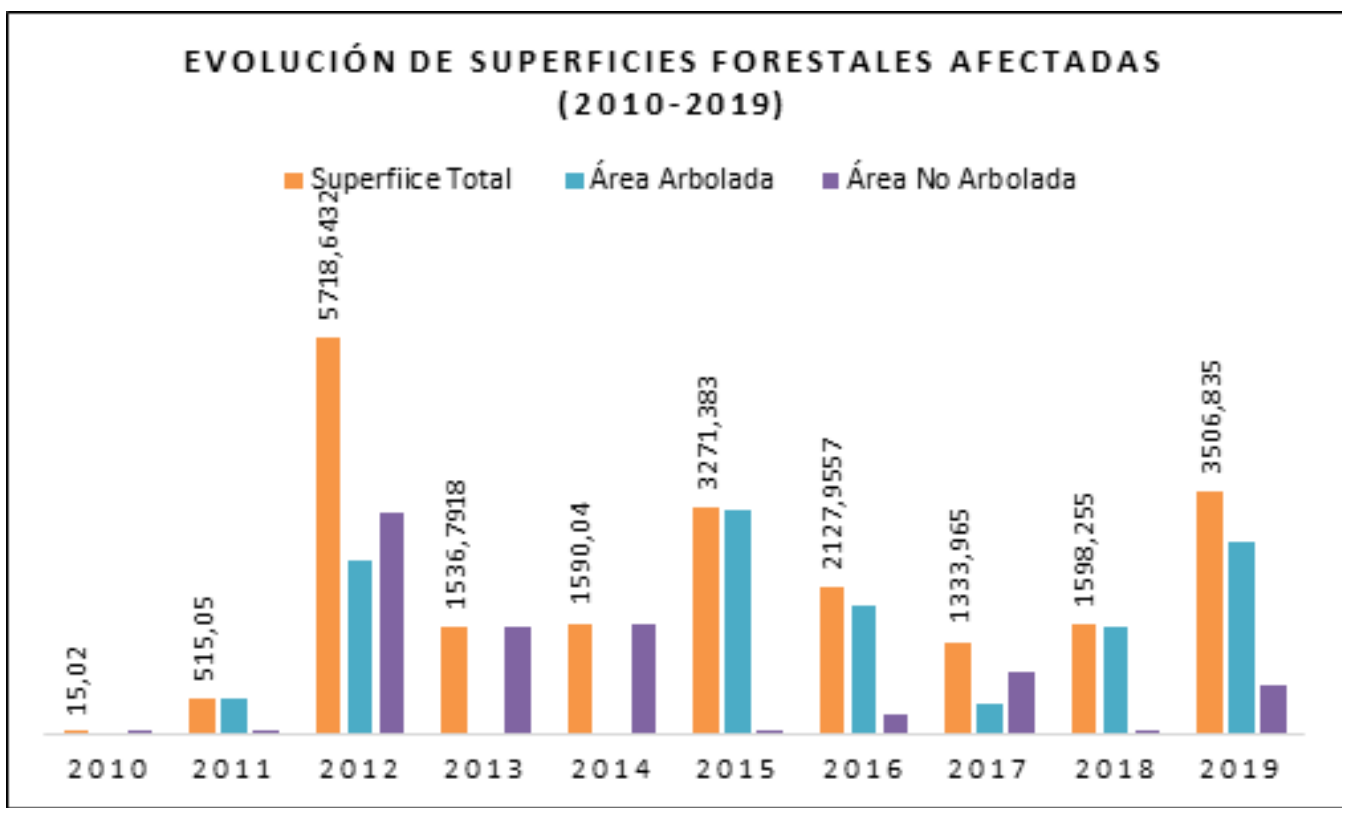

Figura 2. Evolución de superficies forestales afectadas.

El mayor número de incendios forestales en la provincia de Imbabura se registró en el año 2015 (841 incendios forestales), a partir del año 2010 al 2019 se registra un total 2447 incendios forestales reportados según el servicio de gestión de riesgos y emergencias de esta provincia, dejando pérdidas económicas sumamente altas.

Ibarra y Cotacachi son los cantones más afectados en la provincia de Imbabura, teniendo un valor económico total de \$ 679.431,67 por captura de dióxido de carbono 
en el año 2019, debido a bosques y plantaciones de Eucalyptus globulos (especie de mayor abundancia en el sector) las cuales han sido afectadas por los incendios forestales. Estas cifras no reflejan el valor total de las pérdidas ocasionadas por los incendios forestales en el año 2019 en los cantones mencionados en Imbabura, cabe destacar el esfuerzo que se ha ido realizando cada año en los diferentes proyectos de investigación referentes a la valoración socio económica ambiental asociados a los incendios, los cuales presentan una metodología que se aproxima a los valores exactos en cuanto a pérdidas económicas pero hay que mencionar el difícil acceso a las zonas afectadas para la toma de datos.

La mayor parte de la población es consciente que las actividades humanas como el vandalismo y las negligencias son las causas principales por las que se producen los incendios forestales en la provincia de Imbabura, cabe mencionar que las zonas afectadas por incendios forestales en el año 2019 aún no han sido recuperadas en su totalidad. La quema agrícola y de residuos son la segunda actividad humana causante de los incendios forestales, ya que pueden pasar a ser actividades que no se las puede contralar, produciendo así eventos accidentales como incendios.

Los resultados en la producción de biomasa para plantaciones y bosques de Eucalyptus globulos de la presente investigación utilizando la metodología recomendada por el Ministerio de Ambiente son de 29,45 (toneladas/hectárea), resultados que fueron comparados con registros de estudios anteriormente realizados, presentando una similitud en sus valores obtenidos (6).

Es por ello que es importante considerar algunas recomendaciones tales como:

Al aumentar el número de parcelas en la muestra, se obtendrá una mayor precisión y confiabilidad en resultados de biomasa y captura de carbono, así como también los valores económicos generados por los mismos.

Para obtener datos reales con respecto al carbono capturado y biomasa en las plantaciones y bosques afectados por los incendios forestales es necesario monitorear estas variables en las parcelas permanentes.

Es necesario incluir otros cantones y provincias del Ecuador donde se encuentren otras de plantaciones y bosques, ya que no existen investigaciones que reflejen valores exactos con respecto a la valoración socio económica ambiental en el país en zonas con diversa flora y fauna.

\section{CONCLUSIONES}

Continuar con esta investigación para determinar el incremento de biomasa y captura de dióxido de carbono anualmente, determinando así la eficiencia en la implementación de los planes de reforestación, mitigación y prevención ante incendios forestales.

\section{REFERENCIAS BIBLIOGRÁFICAS}

1. Greenpeace. Cada vez más incendios devastadores, ¿Qué está pasando? Greenpeace España. (s/f). Recuperado el 22 de mayo de 2020, de https://es.greenpeace.org/es/noticias/ cada-vez-mas-incendios-devastadores-queesta-pasando/ 
2. Lierop P, V, y Moore PF. Relaciones internacionales para reducir los impactos de los incendios forestales - algo de Historia y algunas reflexiones. Gen. Tech. Rep. PSWGTR-261, (Español). Albany, CA: Departamento de Agricultura de Los EE.UU., Servicio Forestal, Estación de Investigación Del Pacífico Suroeste. 2019:1-16,261-ESP. https://www.fs.usda.gov/ treesearch/pubs/57743

3. Evaluación de los recursos forestales mundiales 2020. FAO. 2020, https://doi. org/10.4060/ca8753es

4. Cancino C, y O, J. Dendrometría básica. Universidad de Concepción. Facultad de
Ciencias Forestales. Departamento Manejo de Bosques y Medio Ambiente. 2012, http:// repositorio.udec.cl/jspui/handle/11594/407

5. Díaz-Hormazábal I, González ME. Análisis espacio-temporal de incendios forestales en la región del Maule, Chile. Bosque (Valdivia), 2016;37(1):147-158. https://doi.org/10.4067/ S0717-92002016000100014

6. Hurtado A, y Edisson F. Determinación del factor de forma de la especie Eucalyptus saligna (Eucalipto) en una plantación forestal comercialdelsectorTambilloBajo,cantónColta, provincia de Chimborazo. 2018, http://dspace. espoch.edu.ec/handle/123456789/10372 